Bull. Austral. Math. Soc.

$65 \mathrm{~J} 15,65$ н 10

VOL. 43 (1991) [51-61]

\title{
ON TIME DEPENDENT MULTISTEP DYNAMIC PROCESSES
}

\author{
Ferenc Szidarovszky and Ioannis K. Argyros
}

The discrete time scale Liapunov theory is extended to time dependent, higher order, nonlinear difference equations in a partially ordered topological space. The monotone convergence of the solution is examined and the speed of convergence is estimated.

\section{INTRODUCTION}

Iteration processes are extremely important in solving optimisation problems, linear and nonlinear equations, and in general, they are used in all fields of applied mathematics.

In the context of nonlinear programming Zangwill [15] presented a general theory on convergence of iteration processes based on point-to-set mappings. He investigated only one-step stationary iterations, and he proved that the process either terminates after a finite number of steps or the limit of any convergent subsequence is a solution. Special but practically useful criteria were derived for example by Brock and Scheinkman [1], Fujimoto [4], [5], Szidarovszky and Okuguchi [10] based on special selections of the Liapunov function.

In this paper the convergence theorem of Zangwill is generalised and extended to nonstationary multistep iteration processes in partially ordered topological spaces. In addition, monotone convergence and the speed of the convergence of the processes are examined.

Before presenting our convergence results, a brief summary of the concepts of special topological spaces is presented.

Let $X$ be a linear space. A subset $K$ of $X$ is called a cone if $K+K \subset K$ and $\alpha K \subset K$ for all $\alpha>0$. The cone $K$ is proper if $K \cap\{-K\}=0$. The relation " $\leqslant$ " defined by

$$
x \leqslant y \text { if and only if } y-x \in K
$$

is a partial ordering on $K$ which is compatible with the linear structure of this space. Two elements $x$ and $y$ of $X$ are called comparable if either $x \leqslant y$ or $y \leqslant x$ holds. The space $X$ endowed with the above relation is called a partially ordered linear space

Received 6 February 1990

Copyright Clearance Centre, Inc. Serial-fee code: 0004-9729/91 $\$ A 2.00+0.00$. 
(POL-space). If $X$ has a topology compatible with its linear structure and if the cone $K$ is closed in that topology then $X$ is called a partially ordered topological space (POTL-space).

We remark that in a POTL-space the intervals $[a, b]=\{x ; a \leqslant x \leqslant b\}$ are closed sets. A lot of examples show that the closedness of the nonnegative cone is not, in general, a strong enough connection between the ordering and the topology (see for example Vandergraft, [13]).

DEFINITION 1: A POTL-space is called normal if, given a local base $U$ for the topology, there exists a positive number $\eta$ such that $0 \leqslant z \in U$ implies $[0, z] \subset \eta U$.

DEFINITION 2: A POTL-space is called regular if every order bounded increasing sequence has a limit.

If the topology of a POTL-space is given by a norm then this space is called a partially ordered normed space (PON-space). If a PON-space is complete with respect to its topology then it is called a partially ordered Banach space(POB-space). According to Definition 1 a PON-space is normal if and only if there exists a positive number $\alpha$ such that

$$
\|x\| \leqslant \alpha\|y\| \text { for all } x, y \in X \text { with } 0 \leqslant x \leqslant y .
$$

Note that any regular POB-space is normal. The reverse is not true. For example, the space $C[0,1]$ of all continuous real functions defined on $[0,1]$, ordered by the cone of nonnegative functions, is normal but is not regular. All finite dimensional spaces are both normal and regular.

\section{Convergence theorems}

Let $S \subset X$ be a set such that $u^{*} \in \bar{S}$, and for $k \geqslant 0$ the point-to-set mappings $f(k ; \cdot)$ are defined on

$$
S^{\ell}=S \times S \times S \cdots \times S,
$$

and for all $t^{(1)}, \ldots, t^{(\ell)} \in S$ and $k \geqslant \ell-1, f\left(k ; t^{(1)}, \ldots, t^{(\ell)}\right)$ is nonempty in $S$. Define the iteration sequence

$$
x_{k+1} \in f\left(k_{k-\ell+1}, x_{k-\ell+2}, \cdots, x_{k}\right)
$$

where $k \geqslant \ell-1, x_{0}, x_{1}, \cdots, x_{\ell-1} \in S$, and an arbitrary element from the set can be selected as the successor of $x_{k}$.

Definition 3: A function $V: S^{\ell} \rightarrow R_{+}$is called the Liapunov function of process (1), if for arbitrary $t^{(i)} \in S\left(i=1,2, \ldots, \ell, t^{(\ell)} \neq u^{*}\right)$ and $y \in f\left(k ; t^{(1)}, \ldots, t^{(\ell)}\right)$ $(k \geqslant \ell-1)$,

$$
V\left(t^{(2)}, \cdots, t^{(\ell)}, y\right)<V\left(t^{(1)}, t^{(2)}, \ldots, t^{(\ell)}\right) .
$$


Definition 4: The Liapunov-function $V$ is called closed, if it is defined on $\bar{S}=$ $\bar{S} \times \bar{S} \times \cdots \times \bar{S}$, furthermore, if $k_{i} \rightarrow \infty, t_{i}^{(j)} \rightarrow t^{(j)^{*}}$

$$
\left(t_{i}^{(j)} \in S \text { for } i \geqslant 0 \text { and } j=1,2, \cdots, \ell, t^{(\ell)^{*}} \neq u^{*}\right),
$$

$y_{i} \in f\left(k_{i} ; t_{i}^{(1)}, \cdots, t_{i}^{(\ell)}\right)(i \geqslant 0)$ and $y_{i} \rightarrow y^{*}$, then

$$
V\left(t^{(2)^{*}}, \cdots, t^{(\ell)^{*}}, y^{*}\right)<V\left(t^{(1)^{*}}, t^{(2)^{*}}, \cdots, t^{(\ell)^{*}}\right)
$$

REMARK. Assume that $f(k ; \cdot) \equiv f(\cdot)$ for all $k, S=\bar{S}$, and mapping $f(\cdot)$ is closed (for the definition of closed mappings see for example Zangwill, [15], p.88), then any Liapunov-function is also closed.

Our main convergence result can be formulated as follows:

THEOREM 1. Assume that $X$ is a topological space and

(A) for all $k \geqslant \ell-1, f\left(k ; t^{(1)}, \ldots, t^{(\ell)}, u^{*}\right)=\left\{u^{*}\right\}$ with arbitrary $t^{(1)}, \cdots, t^{(\ell-1)} \in S$, if $u^{*} \in S \subseteq X$;

(B) the iteration process (1) has a continuous, closed Liapunov function;

(C) there exists a compact set $C$ in $X$ and that for all $k \geqslant \ell-1, x_{k} \in C$. Then $x_{k} \rightarrow u^{*}$ as $k \rightarrow \infty$.

Proof: Condition (A) implies that if for $k \geqslant \ell-1, x_{k}=u^{*}$, then all successors of $x_{k}$ are also equal to $u^{*}$. Hence we may assume that $x_{k} \neq u^{*}(k \geqslant \ell-1)$. Assume that $x_{k} \nrightarrow u^{*}$; then since the sequence is in a compact set there is a subsequence $x_{k_{i}}$ which tends to $x^{*} \neq u^{*}$. The construction of the iteration sequence and the definition of the Liapunov function imply that for all $i \geqslant 0$,

$$
\begin{aligned}
V\left(x_{k_{i+1}-\ell+1}, \cdots, x_{k_{i+1}}\right) & \leqslant V\left(x_{k_{i}-\ell+2}, \cdots, x_{k_{i}}, x_{k_{i}+1}\right) \\
& \leqslant V\left(x_{k_{i}-\ell+1}, \cdots, x_{k_{i}}\right) .
\end{aligned}
$$

Without loss of generality assume that all sequences $\left\{x_{k_{i}-\ell+1}\right\},\left\{x_{i-\ell+2}\right\}, \cdots,\left\{x_{k_{i}}\right\}$, and $\left\{x_{k_{i}+1}\right\}$ are also convergent, otherwise take further subsequences of $\left\{x_{k_{i}}\right\}$. Let $x_{\ell-1}^{*}, \cdots, x_{1}^{*}$ and $y^{*}$ denote the limits of the above subsequences; then the continuity of the Liapunov-function and relation (2) imply that

$$
V\left(x_{\ell-2}^{*}, \cdots, x_{1}^{*}, x^{*}, y^{*}\right)=V\left(x_{\ell-1}^{*}, \cdots, x_{1}^{*}, x^{*}\right)
$$

Since the Liapunov-function is closed, strict inequality must hold in the above relation. This contradiction completes the proof. 
REMARK 1. Assumption $u^{*} \in \bar{S}$ is needed in order to obtain $u^{*}$ as the limit of sequences from $S$. Assumption (A) guarantees that if at any iteration step the solution $u^{*}$ is obtained, then the process remains at the solution. We may also show that the existence of a Liapunov function is not a too strong assumption. Consider the special case when $X$ is a normed space and $f$ is point-to-point from $S$ to $S$, and assume that starting from arbitrary initial sulution $x_{0} \in S$ the process converges to the solution $u^{*}$ of equation $x=f(x)$. Let $V: S \rightarrow R_{+}$be constructed as follows. With selecting $x_{0}=x$ consider sequence $x_{k+1}=f\left(x_{k}\right)(k \geqslant 0)$, and define

$$
V(x)= \begin{cases}0 & \text { if } x=u^{*} \\ \max _{k}\left\|x_{k}-u^{*}\right\| & \text { otherwise }\end{cases}
$$

Obviously $V(f(x)) \leqslant V(x)$ for all $x \in S$. The continuity-type assumptions in (B) are also natural, since without certain continuity conditions no convergence can be established. Assumption (C) is necessarily satisfied, for example, if $x=R^{n}$, and either $S$ is bounded or if for every $B>0$ there exists a $Q>0$ such that $t^{(1)}, \cdots, t^{(\ell)} \in S$ and $\left\|t^{(j)}\right\|>Q$ (for at least one index $j$ ) imply relation

$$
V\left(t^{(1)}, \cdots, t^{(\ell)}\right)>B
$$

In the case of one-step processes (that is, if $\ell=1$ ) this last condition can be reformulated as

$$
\lim _{\substack{\|x\| \rightarrow \infty \\ x \in S}} V(x)=\infty .
$$

REMARK 2. Iteration processes in this general form have real practical importance. Note first that one of the most popular solvers of nonlinear equations is the secant method, which is actually a two-step process. Many dynamic economic processes are based on the selection of optimal strategies by the participants at each time period. If the optimal solution is not unique, then the strategy for the next period can be selected from the set of optimal solutions. Hence the iteration is based on a set-valued mapping. In addition, if the participants' decisions are based on extrapolative expectations on the other's behaviour, then the process becomes multistep. Time dependency of the process follows from price changes, technological development, et cetera. For the description of such models in the oliopoly theory see Okuguchi and Szidarovszky [7].

Assume next that the iteration process is stationary, that is, in recursion (1) function $f$ does not depend on $k$. In this case Theorem 1 reduces to the following.

ThEOREM 2. Assume that $X$ is a topological space, $S \subset X$; furthermore (i) $S=\bar{S}$; 
(ii) for all $t^{(1)}, \ldots, t^{(\ell-1)} \in S$,

$$
f\left(t^{(1)}, \cdots, t^{(\ell-1)}, u^{*}\right)=\left\{u^{*}\right\}
$$

(iii) function $f$ is closed on $S$;

(iv) the iteration process has a continuous Liapunov function;

(v) there exists a compact set $C \subset X$ such that for all $k \geqslant \ell-1, x_{k} \in C$.

Then $x_{k} \rightarrow u^{*}$ as $k \rightarrow \infty$.

REMARK. This result in the special case of $\ell=1$ can be considered as the discretetime-scale counterpart of the famous stability result of Uzawa [12].

\section{MONOTONE CONVERGENCE}

In this section sufficient conditions will be given for the monotone convergence of the iteration scheme (1). Assume now that $X$ is a partially ordered topological space, and $S \subset X$.

DEFINITION 5: The sequence of point-to-set mappings $f(k ; \cdot)$ from $S$ to $S$ is called increasingly isotone on $S$ if for arbitrary $k \geqslant \ell-1, t^{(i)} \in S(i=1,2, \cdots, \ell+1)$ such that $t^{(\ell+1)} \geqslant t^{(\ell)} \geqslant \cdots \geqslant t^{(2)} \geqslant t^{(1)}$ and for any $y_{1} \in f\left(k ; t^{(1)}, \cdots, t^{(\rho)}\right)$ and $y_{2} \in f\left(k+1 ; t^{(2)}, \cdots, t^{(\rho+1)}\right), y_{1} \leqslant y_{2}$.

Definition 6: Point-to-set mapping $f(k ; \cdot): S^{\ell} \rightarrow S$, for a fixed $k(k \geqslant \ell-1)$, is called increasingly isotone if $t^{(i)} \in S(i=1,2, \cdots, \ell+1)$ such that $t^{(\ell+1)} \geqslant t^{(\ell)} \geqslant$ $\cdots \geqslant t^{(2)} \geqslant t^{(1)}$ and $y_{1} \in f\left(k ; t^{(1)}, \ldots, t^{(\ell)}\right)$ and $y_{2} \in f\left(k ; t^{(2)}, \cdots, t^{(\ell+1)}\right)$ imply that $y_{1} \leqslant y_{2}$.

REMARK 1. Note that if $f(k ; \cdot)$ does not depend on $k$, then Definitions 5 and 6 are equivalent.

REMARK 2. In the literature a point-to-set mapping $f(k ; \cdot)$ is called isotone if for all $t^{(i)} \in S, s^{(i)} \in S$ such that $t^{(i)} \leqslant s^{(i)}(i=1,2, \cdots, \ell), y_{1} \leqslant y_{2}$ for all $y_{1} \in$ $f\left(k ; t^{(1)}, \ldots, t^{(\ell)}\right), y_{2} \in f\left(k ; s^{(1)}, \ldots, s^{(\ell)}\right)$. It is obvious that an isotone mapping is increasingly isotone, but the reverse is not necessarily true, as the example of set $S=[0,1] \subset R^{1}$ and function

$$
g\left(t^{(1)} t^{(2)}\right)= \begin{cases}t^{(2)} & \text { if } t^{(1)} \geqslant 2 t^{(2)}-1 \\ t^{(1)}-t^{(2)}+1, & \text { if } t^{(1)}<2 t^{(2)}-1\end{cases}
$$

illustrates. Let the partial order $\leqslant$ be defined as $\left(s^{(1)}, s^{(2)}\right) \leqslant\left(t^{(1)}, t^{(2)}\right)$ if and only if $s^{(1)} \leqslant t^{(1)}$ and $s^{(2)} \leqslant t^{(2)}$. First we show that $g$ is increasingly monotone. 
Select $t^{(1)} \leqslant t^{(2)} \leqslant t^{(3)}$. Note first that $g\left(t^{(1)}, t^{(2)}\right) \leqslant t^{(2)}$. If $t^{(1)} \geqslant 2 t^{(2)}-1$, then $g\left(t^{(1)}, t^{(2)}\right)=t^{(2)} ;$ and if $t^{(1)}<2 t^{(2)}-1$, then

$$
g\left(t^{(1)}, t^{(2)}\right)=t^{(1)}-t^{(2)}+1<2 t^{(2)}-1-t^{(2)}+1=t^{(2)} .
$$

Note next that $g\left(t^{(2)}, t^{(3)}\right) \geqslant t^{(2)}$. If $t^{(2)} \geqslant 2 t^{(3)}-1$, then $g\left(t^{(2)}, t^{(3)}\right)=t^{(3)} \geqslant t^{(2)}$; and if $t^{(2)}<2 t^{(3)}-1$, then

Hence

$$
\begin{aligned}
& g\left(t^{(2)}, t^{(3)}\right)=t^{(2)}-t^{(3)}+1 \geqslant t^{(2)} . \\
& g\left(t^{(1)}, t^{(2)}\right) \leqslant t^{(2)} \leqslant g\left(t^{(2)}, t^{(3)}\right) .
\end{aligned}
$$

We can also verify that the mapping $g$ is not isotone on $S$. Consider points $(t, 1)$ and $(t, 1-\varepsilon)(t, \varepsilon>0 ; t+2 \varepsilon<1)$. Then $g(t, 1)=t$ and $g(t, 1-\varepsilon)=t-(1-\varepsilon)+1=$ $t+\varepsilon>g(t, 1)$. Hence $g$ is not isotone.

THEOREM 3. Assume that in iteration (1) the sequence of mappings $f(k ; \cdot)$ is increasingly isotone; furthermore $x_{i} \in S(0 \leqslant i<\ell-1)$ and $x_{0} \leqslant x_{1} \leqslant \cdots \leqslant x_{\ell-1} \leqslant$ $x_{\ell}$. Then for all $k \geqslant 0, x_{k+1} \geqslant x_{k}$.

Proof: By induction, assume that for $i(i<k), x_{i+1} \geqslant x_{i}$. Then relations $x_{k} \in f\left(k-1, x_{k-\ell}, \cdots, x_{k-1}\right), x_{k+1} \in f\left(k, x_{k-\ell+1}, \cdots, x_{k}\right)$ and the definition of increasingly monotone family of mappings imply that $x_{k} \leqslant x_{k+1}$. Since this inequality holds for $k=0,1, \cdots, \ell-1$, the proof is completed.

Consider next the modified iteration scheme

$$
y_{k+1} \in f\left(k ; y_{k}, y_{k-1}, \cdots, y_{k-\ell+1}\right) \text {. }
$$

Using finite induction, similarly to Theorem 3, we may prove the following:

THEOREM 4. Assume that the sequence of mappings $f(k ; \cdot)$ is increasingly isotone, furthermore $y_{i} \in S(0 \leqslant i \leqslant \ell-1)$ and

$$
y_{0} \geqslant y_{1} \geqslant \cdots \geqslant y_{\ell-1} \geqslant y_{\ell} .
$$

Then for all $k \geqslant 0, y_{k+1} \geqslant y_{k}$.

Corollary. Assume that $X=R^{n}$ and for $k \rightarrow \infty$, the sequences $\left\{x_{k}\right\}$ and $\left\{y_{k}\right\}$ have the same limit $u^{*}$, and $\leqslant$ is the usual partial order of vectors. (That is, $a=a(i) \leqslant b=b(i)$ if and only if $a(i) \leqslant b(i)$ for all i.) Under the conditions of Theorems 3 and 4 , for all $k \geqslant 0$,

$$
x_{k} \leqslant u^{*} \leqslant y_{k}
$$


This relation is very useful in the error analysis of the iteration methods (1) and (3), since for all coordinates $x_{k}(i), y_{k}(i)$ and $u^{*}(i)$ of vectors $x_{k}, y_{k}, u^{*}$, respectively,

and

$$
0 \leqslant u^{*}(i)-x_{k}(i) \leqslant y_{k}(i)-x_{k}(i)
$$

$$
0 \leqslant y_{k}(i)-u^{*}(i) \leqslant y_{k}(i)-x_{k}(i) .
$$

Furthermore, we can show:

Theorem 5. Assume that $X$ is a regular POB-space, $S \subset X$ and

(A) the sequence of mappings $f(k ; \cdot)$ is increasingly isotone in iteration (1) with $x_{i} \in S(0 \leqslant i \leqslant \ell-1)$ and

$$
x_{0} \leqslant x_{1} \leqslant \cdots \leqslant x_{\ell-1} \leqslant x_{\ell}
$$

(B) there exists a set $H_{1}$ defined by $H_{1}=\left\{x \in S ; x \leqslant x_{0}\right\}$ with the property that if for any points $t^{(1)}, t^{(2)}, \cdots, t^{(\ell)}$ in $H_{1}$ with

$$
t^{(1)} \leqslant t^{(2)} \leqslant \cdots \leqslant t^{(\ell)} \leqslant x_{0},
$$

then

$x_{k+1} \leqslant x_{0}$ for any $x_{k+1} \in f\left(k ; t^{(1)}, t^{(2)}, \ldots, t^{(\ell)}\right), k \geqslant \ell-1$.

Then the sequence $\left\{x_{n}\right\}, n \geqslant 0$ generated by the iteration (1) process (1) is monotonically increasing, remains in $H_{1}$ and converges to some $u^{*} \in H_{1}$.

Proof: From (A) and Theorem 3 it follows that the sequence $\left\{x_{n}\right\}, n \geqslant 0$ is monotonically increasing, whereas from $(B)$ we get that the sequence is bounded above by $x_{0}$. Since $X$ is a regular POB-space the sequence $\left\{x_{n}\right\}, n \geqslant 0$, converges to some $u^{*}$ with $u^{*} \leqslant x_{0}$. Hence $u^{*} \in H_{1}$.

That completes the proof of the theorem.

These monotonic properties of the iteration processes are very useful, but in cases where the convergence is very slow the above methods have only very limited practical importance. In the next section of this paper the convergence speed of the above iteration schemes is estimated and practical error estimates are derived.

\section{Estimates on the SPEED OF CONVERGENCE}

We can now formulate the following theorem.

Theorem 6. Assume that $X$ is a normal POB-space, $S \subset X$ and

(A) the sequence of mappings $f(k ; \cdot)$ is increasing isotone in iteration (1) with $x_{i} \in S(0 \leqslant i \leqslant \ell-1)$ and $x_{0} \leqslant x_{1} \leqslant \cdots \leqslant x_{\ell-1} \leqslant x_{\ell} ;$

(B) There exists a constant $b$ with $0 \leqslant b<1$ such that

$$
x_{n+2}-x_{n+1} \leqslant b\left(x_{n+1}-x_{n}\right) \text {, for all } n \geqslant 0 .
$$


Then the sequence $\left\{x_{n}\right\}, n \geqslant 0$, generated by the iteration process (1) is monotonically increasing and converges to some $u^{*}$ with

$$
\left\|x_{n}-u^{*}\right\| \leqslant \frac{\alpha\left\|x_{1}-x_{0}\right\|_{1}}{1-b} b^{n}, \quad n \geqslant 0 .
$$

Proof: From (A) and Theorem 3 it follows that the sequence $\left\{x_{n}\right\}$ is monotonically increasing and inequality (4) can be rewritten as

$$
0 \leqslant x_{n+2}-x_{n+1} \leqslant b\left(x_{n+1}-x_{n}\right), \quad n \geqslant 0 .
$$

Using the above inequality we get

$$
0 \leqslant x_{n+p}-x_{n}=\sum_{i=0}^{p-1}\left(x_{n+i+1}-x_{n+i}\right) \leqslant \frac{x_{1}-x_{0}}{1-b} b^{n}, \quad p \geqslant 0 .
$$

Since $X$ is normal we deduce

$$
\left\|x_{n+p}-x_{n}\right\| \leqslant \alpha \frac{\left\|x_{1}-x_{0}\right\|_{1}}{1-b} b^{n} .
$$

It nor follows that the sequence $\left\{x_{n}\right\}, n \geqslant 0$, is a Cauchy sequence in a Banach space and as such it converges to some $u^{*}$. By letting $p \rightarrow \infty$ we obtain (5).

That completes the proof of the theorem.

Note that an identical theorem can be proved if the assumptions (A) and (B) in the above theorem are replaced with the condition

$$
0 \leqslant x_{n+2}-x_{n+1} \leqslant b\left(x_{n+1}-x_{n}\right),
$$

for all $n \geqslant 0$ and some $b, 0 \leqslant b<1$. Let us define the set $H_{2}$ by $H_{2}=\left\{x \in S ; x_{0} \leqslant\right.$ $\left.x,\left\|x-x_{0}\right\| \leqslant h\right\}$ for some $h>0$.

Then we can show the following theorem.

THEOREM 7. Let $X$ be a normal POB-space, $S \subset X$ and assume that the following conditions are satisfied:

$$
\begin{gathered}
\alpha \frac{\left\|x_{1}-x_{0}\right\|}{1-c_{2}} \leqslant h, \\
c_{1}\left(x_{n}-x_{n-1}\right) \leqslant x_{n+1}-x_{n} \leqslant c_{2}\left(x_{n}-x_{n-1}\right), n \geqslant 1,0 \leqslant c_{1} \leqslant c_{2}<1, \\
x_{0} \leqslant x_{n}, \\
\left\|x_{n}-x_{0}\right\| \leqslant h
\end{gathered}
$$


and

$$
0 \leqslant x_{n+1}-x_{n} \leqslant c_{2}^{n}\left(x_{1}-x_{0}\right) \text { for all } n=0,1,2, \ldots, \ell-1
$$

Then the sequence $\left\{x_{n}\right\}, n \geqslant 0$, generated by the iteration process (1) is monotonically increasing and converges to some $u^{*}$ with

$$
\left\|x_{n}-u^{*}\right\| \leqslant \alpha \frac{\left\|x_{1}-x_{0}\right\|}{1-c_{2}} c_{2}^{n} \text { for all } n \geqslant 0 .
$$

Proof: We will show that the estimates (8), (9) and (10) are true for all $n \geqslant 0$. For $n=0,1,2, \cdots, \ell-1$, they hold by hypothesis. Let us suppose that they are true for $n=0,1,2, \ldots, k$ with $k \geqslant \ell-1$. From (8) and (10) for $n=k$ it follows that

$$
x_{0} \leqslant x_{k} \leqslant x_{k+1}
$$

and thus (8) is true for $n=k+1$.

Using (10), the above inequality, and the properties of the partial order $\leqslant$, we have successively:

$$
0 \leqslant x_{k+1}-x_{0}=\sum_{i=0}^{k}\left(x_{i+1}-x_{i}\right) \leqslant\left(x_{1}-x_{0}\right) \sum_{i=1}^{k} c_{2}^{i} \leqslant \frac{x_{1}-x_{0}}{1-c_{2}}
$$

where from (6) we deduce that (9) is true for $n=k+1$.

From (7), (10) and the induction hypothesis we get

$$
0 \leqslant x_{k+2}-x_{k+1} \leqslant c_{2} c_{2}^{k}\left(x_{1}-x_{0}\right)=c_{2}^{k+1}\left(x_{1}-x_{0}\right) .
$$

It now follows that (10) is true for $n=k+1$. Moreover for $p \geqslant 0$ we get

$$
0 \leqslant x_{n+p}-x_{n}=\sum_{i=0}^{p-1}\left(x_{n+i+1}-x_{n+i}\right) \leqslant \frac{x_{1}-x_{0}}{1-c_{2}} c_{2}^{n}
$$

from which we obtain

$$
\left\|x_{n+p}-x_{n}\right\| \leqslant \alpha \frac{\left\|x_{1}-x_{0}\right\|}{1-c_{2}} c_{2}^{n} \leqslant h c_{2}^{n} .
$$

The above inequality shows that the sequence $\left\{x_{n}\right\}, n \geqslant 0$ is Cauchy in a POB-space and as such it converges to some $u^{*}$. By letting $p \rightarrow \infty$ in (12) we obtain (11).

That completes the proof of the theorem. 
REMARK 1. Note that a similar theorem can be proved if the condition (7) is replaced by the relation

$$
0 \leqslant x_{n+1}-x_{n} \leqslant c_{2}\left(x_{n}-x_{n-1}\right), n \geqslant 1,0 \leqslant c_{2}<1 .
$$

REMARK 2. Assume that there exists a sequence $c_{2 n}^{(n)}, n \geqslant 0$, such that more generally

$$
0 \leqslant x_{n+1}-x_{n} \leqslant c_{2}^{(n)}\left(x_{n}-x_{n-1}\right), n \geqslant 1,0 \leqslant c_{2}^{(n)} \leqslant q<1 .
$$

Then similarly to (12) we have that

where

$$
\begin{aligned}
x_{n+p}-x_{n} & =\sum_{i=0}^{p-1}\left(x_{n+i+1}-x_{n+i}\right) \\
& \leqslant\left(x_{1}-x_{0}\right)\left(v_{n}+v_{n+1}+\cdots+v_{n+p-1}\right)
\end{aligned}
$$

Hence

$$
\begin{gathered}
v_{n}=c_{2}^{(1)} c_{2}^{(2)} \cdots c_{2}^{(n)} . \\
x_{n+p}-x_{n} \leqslant\left(x_{1}-x_{0}\right) v_{n}\left(1+q+q^{2}+\cdots\right)
\end{gathered}
$$

$$
=\frac{x_{1}-x_{0}}{1-q} v_{n}
$$

and therefore (11) is modified as

$$
\left\|x_{n}-u^{*}\right\| \leqslant \alpha \frac{\left\|x_{1}-x_{0}\right\|}{1-q} v_{n} .
$$

In the special case when $c_{2}^{(n)}$ is a decreasing sequence we may select $q=c_{2}^{(1)}$.

\section{REFERENCES}

[1] W.A. Brock and J.A. Scheinkman, 'Some results on global asymptotic stability of difference equations', J. Econom. Theory 10 (1975), 265-268.

[2] P. Chander, 'The nonlinear input-output model', J. Econom. Theory 30 (1983), 219-229.

[3] M.J. Chien and L. Chan, 'Nonlinear input-output model with piecewise affice coefficients', J. Econom. Theory 21 (1979), 389-410.

[4] T. Fujimoto, 'Asymptotic stability of non-linear difference equations', Econom. Lett. 22 (1987), 247-250.

[5] T. Fujimoto, 'Global asymptotic stability of non-linear difference equations II', Econom. Lett. 23 (1987), 275-277.

[6] K. Okuguchi and F. Szidarovszky, 'On a nonlinear input-output system', Math. Social Sci. 13 (1987), 277-281.

[7] K. Okuguchi and F. Szidarovszky (to appear), The Theory of Oliogopoly with Multi-Product Firms (Springer-Verlay, New York, Heidelberg, Berlin, 1990 to appear). 
[8] J.M. Ortega and W.C. Rheinboldt, Iterative Solutions of Non-Linear Equations in Several Varibles (Academic Press, New York, London, 1970).

[9] R. Redheffer and W. Walter, 'A comparison theorem for difference inequalities', $J$. of Differential Equations 44 (1982), 111-117.

[10] F. Szidarovszky and K. Okuguchi, 'A note on global asymptotic stability of non-linear difference equations', Econom. Lett. 26 (1988), 349-352.

[11] F. Szidarovszky and S. Yakowitz, Principles and Procedures of Numerical Analysis (Plenum Press, New York, London, 1978).

[12] H. Uzawa, 'The Stability of Dynamic Processes', Econometrica 29 (1961), 617-631.

[13] J.S. Vandergraft, 'Newton's method for convex operators in partially ordered spaces', SIAM J. Numer. Anal. 4 (1967), 406-432.

[14] H.K. Wilson, Ordinary Differential Equations (Addison Wesley, Reading, Mass., 1971).

[15] W.I. Zangwill, Nonlinear Programming. A Unified Approach (Prentice-Hall, Englewood Cliffs, N.J., 1969).

Department of Systems and Industrial Engineering

University of Arizona

Tucson, AZ 85721

United States of America
Department of Mathematics Cameron University

Lawton OK 73505-6377

United States of America 